\title{
Flebitis infecciosa o no infecciosa: lecciones de un programa intervencional sobre flebitis asociada a catéter venoso periférico
}

\author{
Teresa Vergara, Elena Véliz, Alberto Fica y Jordan Leiva
}

\section{Infectious or noninfectious phlebitis: lessons from a an interventional programm on phlebitis associated to peripheral venous catheter}

Background: There is no consensus definition to distinguish infectious from non-infectious phlebitis associated to peripheral venous catheter. Aims: To evaluate the impact of an intervention program on the rate of infectious (those associated to bacteremia or local purulent discharge) and non-infectious phlebitis (the rest) and identify differential features. Methods: Interventional study developed in three stages: basal measurement, intervention, and evaluation. Results: Ten infectious and 186 non-infectious phlebitis were registered. Infectious phlebitis diminished after intervention ( 0.2 to 0.04 events per 1,000 bed-days; $p=0.02)$ but not the rest $(2.3$ per 1,000 bed-days before and after). Five of 10 patients with infectious phlebitis had bacteremia, one with infectious endocarditis and valve replacement, and other with septic shock and a fatal outcome. None of the patients in the non-infectious group presented complications. Infectious phlebitis appeared later (mean 4.1 versus 2.4 days; $p=0.007$ ) and were associated to fever $(40 \%$ vs $5.9 \%, \mathrm{p}=0.004)$. Non-infectious phlebitis was associated to irritating compounds (OR 6.1; $\left.\mathrm{IC}_{95} 1.3-29, \mathrm{p}<0.05\right)$. Conclusions: The intervention program demonstrated a favorable impact only on the rate of infectious phlebitis. Distinction appears to be relevant because those of infectious origin are associated with fever, complications or death, respond to an intervention program, and emerge lately.

Key words: Peripheral venous catheterization; phlebitis; bacteremia; nosocomial infection; intervention study.

Palabras clave: Catéter venoso periférico, flebitis, bacteriemia, infección nosocomial, estudio de intervención.

\section{Introducción}

\section{$\mathrm{L}$}

os catéteres venosos periféricos (CVP) constituyen una parte esencial del trabajo clínico y son usados para administrar fluidos, nutrientes, fármacos $\mathrm{o}$ hemoderivados. Los CVP pueden asociarse a complicaciones locales o sistémicas como flebitis, bacteriemias o endocarditis lo que aumenta la morbilidad o mortalidad durante la hospitalización ${ }^{1}$. Los eventos de flebitis asociados a CVP han sido clasificados de acuerdo a su gravedad pero no existe una clara distinción disponible en la literatura médica respecto a su potencial origen irritativo, químico o traumático por una parte, o infeccioso, exceptuando aquellos casos asociados a bacteriemia sin otra causa evidente ${ }^{2,3}$. Esta frontera imprecisa hace difícil identificar factores ligados a su origen y en forma más relevante, evaluar el impacto de los programas de intervención en la tasa de flebitis.

Las tasas de flebitis asociada a CVP son variables, con algunos estudios que indican valores de 2,5 a 12,9 eventos por 1.000 días $^{4,5}$. Sin embargo, en África algunos estudios observacionales han revelado una prevalencia cercana al $60 \%{ }^{6}$.

Los programas de prevención de flebitis asociada a CVP han logrado reducir su frecuencia global ${ }^{3}$, pero no se sabe en qué proporción aquellas de origen irritativo, químico o traumático responden a una intervención dada, en contraste con aquellas de naturaleza infecciosa, simplemente porque no existen parámetros disponibles para distinguirlas. Las bacteriemias están en un extremo de las flebitis infecciosas, fáciles de reconocer, pero probablemente sólo constituyen una fracción de las de tipo infeccioso. Se han elaborado recomendaciones para prevenir las flebitis infecciosas ${ }^{7}$, pero estas medidas podrían ser inefectivas, si la mayoría de las flebitis en la institución son de causa no infecciosa.

El objetivo de este estudio fue evaluar el impacto de un programa de intervención en la frecuencia global de flebitis asociada a CVP en un hospital general de Chile. Además, aplicamos criterios definidos localmente para reconocer eventos infecciosos y evaluamos si el programa de intervención tenía algún efecto sobre las flebitis de diferente naturaleza.

\section{Pacientes y Métodos}

\section{Escenario y diseño del estudio}

Este trabajo fue desarrollado en el Hospital Militar de Santiago, un centro de alta complejidad que atiende personal militar activo y en retiro, sus cargas familiares
Hospital Militar de Santiago. Unidad de Infecciones Asociadas a la Atención de Salud, Chile (TV, EV, AF).

Servicio de Farmacia (JL).

Conflicto de intereses: No Financiamiento: Ninguno.

Recibido: 10 de enero de 2017 Aceptado: 6 de julio de 2017

Correspondencia a: Teresa Vergara Messina tvergara@hms.cl 
y también población civil general. El programa de intervención fue aplicado con el objetivo de disminuir la frecuencia global de flebitis asociada a CVP y la de flebitis infecciosa, mediante un diseño antes-después. El estudio se ejecutó en tres etapas en pacientes adultos y pediátricos hospitalizados, excluyendo los Servicios de Psiquiatría y Ginecología/Obstetricia. Las etapas específicas correspondieron a la etapa diagnóstica: de julio a diciembre de 2014 (6 meses), etapa de intervención de enero a abril de 2015 (4 meses) y la etapa de evaluación de mayo a diciembre de 2015 (8 meses). La vigilancia de los eventos de flebitis asociada a CVP fue mantenida durante todo el estudio en forma pasiva desde diferentes servicios hacia la Unidad de Infecciones Asociadas a la Atención de Salud (IAAS).

\section{Etapa diagnóstica y definición de flebitis infecciosa y no infecciosa}

En esta etapa, profesionales de enfermería reportaron diariamente los eventos de flebitis a la Unidad de IAAS. Las flebitis por compuestos irritantes, química o traumática fueron definidas por la presencia de eritema, edema, induración o presencia de un cordón venoso palpable en el sitio del CVP. Las flebitis infecciosas fueron arbitrariamente definidas ante la presencia de pus en el sitio de inserción o bacteriemia sin otro foco evidenciable. La asignación como flebitis no infecciosa o infecciosa fue realizada por un profesional de la Unidad IAAS luego de analizar los resultados de los hemocultivos, otras fuentes potenciales y sus características clínicas. La frecuencia de flebitis en esta etapa y en la de evaluación fue expresada como eventos por 1.000 días camas ocupadas.

\section{Etapa de intervención}

Durante esta etapa se aplicaron diferentes actividades disponibles en la literatura científica ${ }^{7}$. Estas estrategias incluyeron capacitación, supervisión y uso de insumos seleccionados. Las sesiones de capacitación fueron desarrolladas por el personal de la Unidad de IAAS durante $10 \mathrm{~min}$ a profesionales de enfermería al término de los turnos nocturnos. Para ello se hizo un paso demostrativo con un set de CVP, llave de tres pasos, alargador venoso y conector sin aguja, enfatizando su uso y mantención apropiada. Se incluyó específicamente la exigencia de un apósito semipermeable, seco y bien adherido ${ }^{8,9}$, el apósito no debía impedir la observación del sitio de inserción y además se recomendó el uso de un alargador venoso para disminuir el trauma en el sitio de entrada ${ }^{10}$. También se incluyó un refuerzo sobre la desinfección de los puertos de entrada y del conector sin aguja e higiene de manos antes de su manipulación (Figuras 1 y 2) ${ }^{7}$. La supervisión fue efectuada tres veces por semana con retroalimentación inmediata a los profesionales de enfermería. La pauta monitorizó la presencia de un apósito seco, limpio y bien adherido, alargador venoso presente y ausencia de sangre visible en el circuito venoso. Se permitió la aplicación de conectores sin aguja sólo en el caso de administración intermitente de fluidos o medicamentos. Los conectores sin aguja permitidos requerían la presencia de un tabique interno septado, que no impidiera el flujo laminar y superficies lisas que permitieran una desinfección eficiente y transparentes para la observación de residuo sanguíneo en su interior. Estas características se han asociado a una reducción en el riesgo de complicaciones infecciosas ${ }^{11-15}$.

\section{Etapa de evaluación}

Durante esta etapa no se hicieron actividades de capacitación, se mantuvo el sistema de vigilancia pasiva y se calculó la frecuencia de flebitis como se describió previamente. Además, se efectuó una medición de la adherencia de las medidas exigidas, las que fueron comparadas con datos similares obtenidos en un estudio observacional el año 2013.

\section{Caracterización, desenlaces y análisis de eventos letales de flebitis}

Los eventos de flebitis fueron analizados de acuerdo a su momento de detección respecto a la instalación del

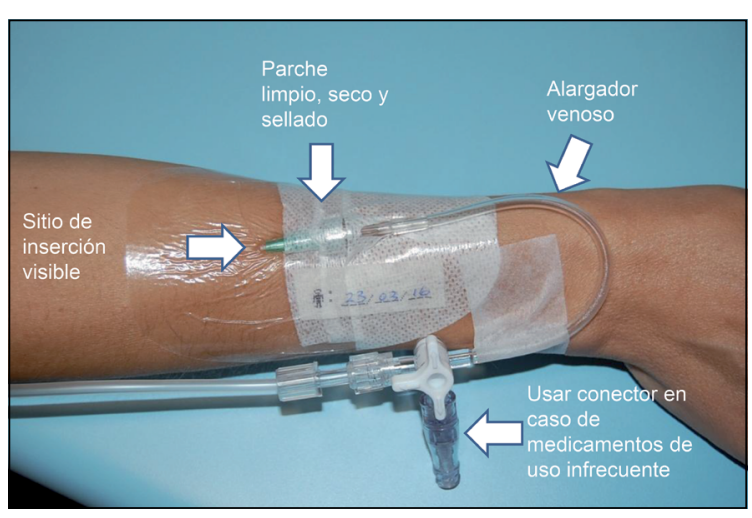

Figura 1.

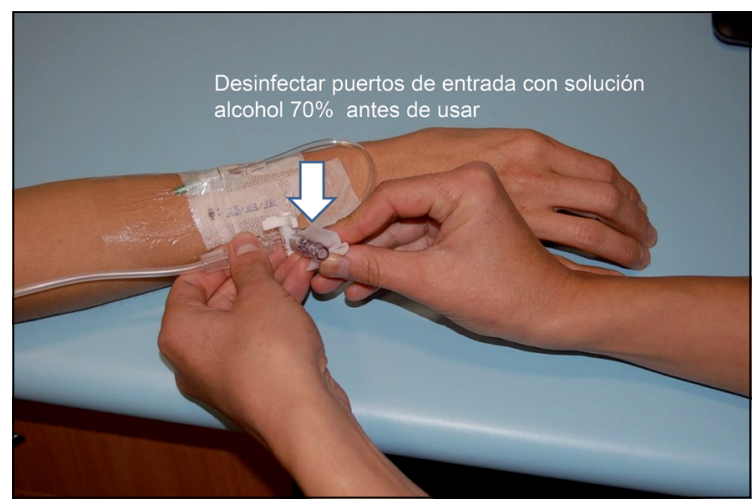

Figura 2. 
CVP, presencia de hipotensión arterial diastólica (al menos dos registros $<60 \mathrm{mmHg}$ el mismo día), fiebre (temperatura $>37^{\circ} \mathrm{C}$ axilar) y solicitud de hemocultivos y sus resultados. El desenlace (fallecido o vivo) fue evaluado a los 14 días de la fecha del diagnóstico de flebitis. Se efectuó un análisis para ver si la flebitis infecciosa provocó directamente la muerte o no en los casos con un desenlace fatal.

\section{Uso concomitante de compuestos irritantes}

Los medicamentos aplicados a través del CVP fueron clasificados como irritantes o no irritantes de acuerdo a su $\mathrm{pH}$ y osmolaridad (irritante si $\mathrm{pH}<5,0 \mathrm{u}$ osmolaridad $>$ 3 veces la del plasma) y datos de la literatura médica ${ }^{16-20}$.

\section{Análisis estadístico}

La asociación de variables categóricas tales como fiebre e hipotensión arterial diastólica con flebitis infecciosa, fue explorada mediante tablas de contingencia y cálculo de la razón de disparidad (Odds Ratio, OR). Las diferencias en el tiempo de aparición de flebitis infecciosa o no infecciosa fueron analizadas por la prueba de Mann Whitney. La asociación de uso de compuestos irritantes con flebitis no infecciosa fue estudiada mediante tablas de contingencia y la prueba bilateral de Fisher. El impacto del programa de intervención fue analizado con tablas de contingencia, usando la frecuencia absoluta, antes y después de la intervención y el respectivo cálculo OR.

\section{Resultados}

Durante las fases de diagnóstico (antes) y evaluación, se registraron 196 eventos de flebitis asociada a CVP: 10 de origen infeccioso y 186 no infeccioso. Estos casos fueron observados en salas de medicina adultos (n: 83;
42,3\%) o quirúrgicas (n: $67 ; 34,2 \%$ ) y el resto en Unidades Críticas (n: $31 ; 15,8 \%)$, quimioterapia $(n=6 ; 3,1 \%)$, Pediatría (n: $5 ; 2,6 \%$ ) u otros servicios (n: $4 ; 2 \%$ ).

La mayor parte de los pacientes experimentó sólo un evento de flebitis no infecciosa (n: 177; 90,3\%) pero 19 presentaron más de 1: 16 con dos eventos $(8,2 \%), 2$ con tres eventos $(1 \%)$ y 1 con cuatro $(0,5 \%)$.

La edad promedio de los 177 pacientes afectados fue 66,4 años (rango 1 a 95). Seis casos fueron observados en niños $(3,4 \%)$.

Las flebitis infecciosas fueron diagnosticadas por bacteriemia en 5 casos y en los otros 5 por la presencia de pus en el sitio de inserción (Tabla 1). Las infecciones del torrente sanguíneo estuvieron asociadas a diferentes especies del género Staphylococcus (S. aureus, S. capitis y $S$. haemolyticus) y dos especies de bacilos gramnegativos en una infección polimicrobiana (Klebsiella pneumoniae/ Citrobacter freundii). Los casos asociados a Staphylococcus coagulasa negativa tuvieron al menos dos muestras independientes positivas, descartando contaminación. No se tomaron cultivos en los casos detectados por pus. Ocho de 10 eventos fueron reportados en pacientes adultos y seis tenían co-morbilidades. Los dos casos asociados a $S$. aureus evolucionaron con complicaciones: uno con endocarditis infecciosa que requirió reemplazo valvular y otro con un shock séptico fatal. Este último, fue el único paciente que tuvo un desenlace adverso (Tabla 1). Los eventos de flebitis, fuesen infecciosos o no infecciosos, aparecieron en un promedio de 2,5 días después de la instalación del CVP (rango 0 a 17 días).

La fiebre estuvo presente el día del diagnóstico en 15 eventos de flebitis $(7,7 \%)$ y 20 eventos tuvieron hipotensión arterial diastólica (10,3\%). Se tomaron hemocultivos en 29 eventos (14,8\%). Los casos con hipotensión arterial diastólica se distribuyeron sin diferencias significativas entre los eventos infecciosos y no infecciosos (datos no

\begin{tabular}{|c|c|c|c|c|c|c|}
\hline Caso & $\begin{array}{l}\text { Edad } \\
\text { (años) }\end{array}$ & Género & Criterio definición/microorganismo & Co-morbilidad & $\begin{array}{c}\text { Complicaciones asociadas } \\
\text { a flebitis }\end{array}$ & Desenlace \\
\hline 1 & 53 & M & Bacteriemia/S. aureus & Ninguna & Endocarditis & Reemplazo valvular - Vivo \\
\hline 2 & 80 & M & Bacteriemia/S. capitis* & Leucemia & Ninguna & Vivo \\
\hline 3 & 2 & M & Bacteriemia/K. pneumoniae - C. freundii & Ninguna & Ninguna & Vivo \\
\hline 4 & 66 & $\mathrm{~F}$ & Pus en el sitio de inserción & DM & Ninguna & Vivo \\
\hline 5 & 54 & $\mathrm{~F}$ & Pus en el sitio de inserción & Cáncer & Ninguna & Vivo \\
\hline 6 & 5 & M & Pus en el sitio de inserción & Ninguna & Ninguna & Vivo \\
\hline 7 & 65 & $\mathrm{~F}$ & Pus en el sitio de inserción & Falla cardíaca/DHC & Ninguna & Vivo \\
\hline 8 & 68 & M & Pus en el sitio de inserción & DM/falla renal & Ninguna & Vivo \\
\hline 9 & 59 & $\mathrm{~F}$ & Bacteriemia/S. haemolyticus* & Ninguna & Ninguna & Vivo \\
\hline 10 & 86 & M & Bacteriemia/S. aureus & VIH/SIDA & Shock séptico & Fallece \\
\hline
\end{tabular}


mostrados). En contraste, cuatro de 10 eventos de flebitis infecciosa tuvieron fiebre (40\%) versus 11 de 186 del grupo no infeccioso (5,9\%) (OR 10,6; IC 95\% 2,6 a $43,2 ; \mathrm{p}=0,004)$. Además, sólo tres eventos presentaron simultáneamente fiebre e hipotensión arterial diastólica $(1,5 \%)$. Esta característica estuvo significativamente asociada a un origen infeccioso (dos de 10 casos infecciosos presentaban esta combinación versus uno de 185 eventos no infecciosos con datos completos disponibles, OR 46 IC

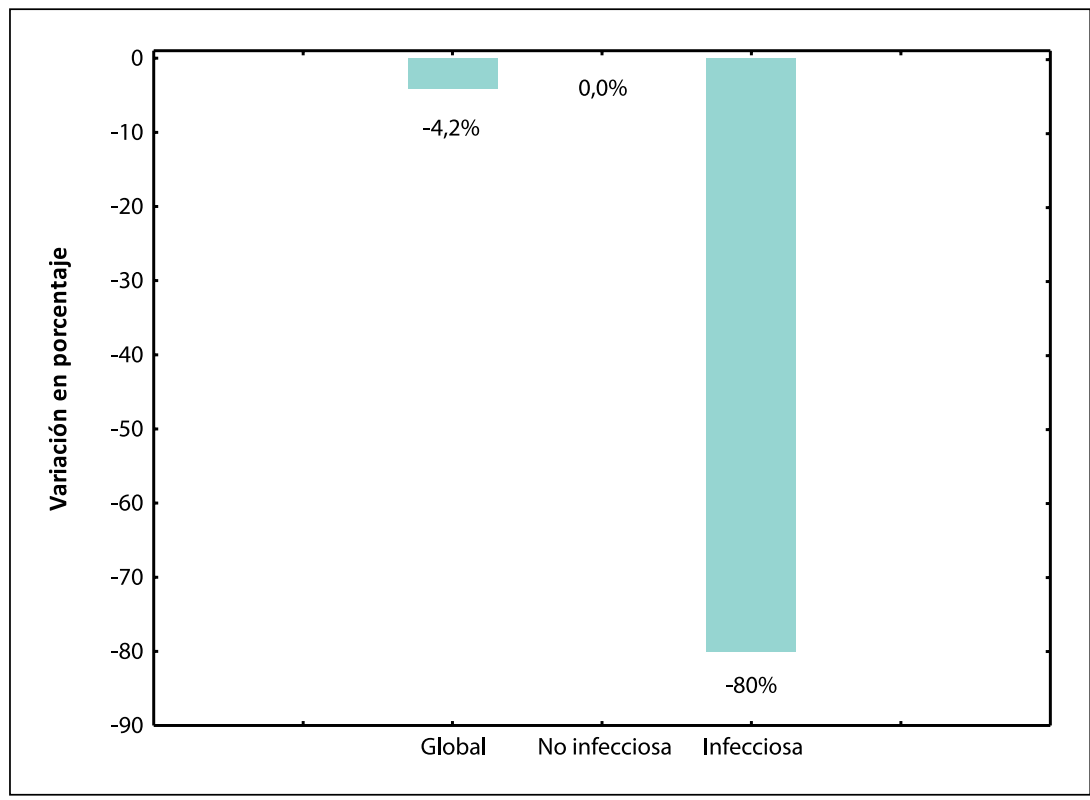

Figura 3. Variación en la densidad de casos de flebitis con el programa de intervención.

Tabla 2. Distribución de eventos de flebitis antes y después del Programa de Intervención

\begin{tabular}{lccc} 
& Flebitis infecciosa & No infecciosa & Total \\
\hline Post intervención & 2 & $\mathbf{n}$ & 114 \\
Basal & 8 & 112 & 82 \\
Total & 10 & 74 & 196
\end{tabular}

Tabla 3. Cumplimiento de las medidas preventivas de flebitis asociada a CVP durante los años 2013 y 2015

\begin{tabular}{|c|c|c|}
\hline Parámetro & $\begin{array}{c}2013 \\
\%\end{array}$ & $\begin{array}{c}2015 \\
\%\end{array}$ \\
\hline Apósito en condiciones apropiadas & 81,4 & 88,9 \\
\hline CVP justificado & 77,5 & 88,9 \\
\hline Set de infusión limpio & 70,5 & 84,4 \\
\hline Registro escrito de existencia de CVP & $70,6^{*}$ & $95,1 *$ \\
\hline Todos los anteriores & 45,1 ** & $70,4^{* *}$ \\
\hline
\end{tabular}

${ }^{*} p=0,0001 ;{ }^{* *} p=0,0108$ por diferencia de proporciones. CVP: catéter venoso periférico.
95\% 3,8 a 561; $p=0,007)$. Más aún, la flebitis infecciosa se desarrolló más tardíamente que la no infecciosa $(4,1$ versus 2,4 días, respectivamente; $p=0,007$ por prueba de Mann Whitney). Ninguno de los pacientes del grupo de flebitis no infecciosa murió en las dos semanas siguientes después del diagnóstico, en contraste con uno del grupo infeccioso.

Ocho de 10 eventos con flebitis infecciosa $(80 \%)$ fueron tratados con antimicrobianos debido a esta complicación y en dos los eventos en los que éstos no fueron usados, la flebitis sólo se asoció a pus local. En contraste, sólo cuatro de 186 eventos no infecciosos $(2,2 \%)$ recibieron antimicrobianos, el que en todos los casos fue por vía oral (flucloxacilina en tres y cotrimoxazol en uno). No observamos diferencias en edad, género o sala de hospitalización entre los grupos de flebitis.

\section{Uso de compuestos irritantes parenterales}

Analizamos la administración de compuestos irritantes a través del CVP en cada paciente que desarrolló flebitis. En 26 casos no se utilizaron fármacos, nutrición parenteral o transfusiones a través del CVP antes de la aparición de la flebitis $(13,3 \%)$ y tres de los 10 eventos infecciosos estaban en este subgrupo. Ciento setenta eventos estuvieron asociados a la administración de cualquier tipo de tratamiento por el CVP y en este subgrupo, la aplicación de compuestos irritantes estuvo significativamente asociada al desarrollo de flebitis no infecciosa: 134 de 163 eventos del grupo no infeccioso recibió algún compuesto irritante antes de la flebitis $(82,2 \%)$; en contraste, sólo tres de siete del grupo infeccioso (43\%) recibieron al menos un compuesto irritante antes (OR 6,1 IC 95 1,3-29; p <0,05).

\section{Impacto del Programa de Intervención}

Durante la etapa diagnóstica, la tasa global de flebitis fue de 2,4 por 1.000 camas ocupadas. Durante la etapa de evaluación, la tasa global no cambió en forma importante (2,4 a 2,3 por 1.000 camas ocupadas, $-4,2 \%$ ). Sin embargo, al comparar ambos períodos, se apreció una reducción en la tasa de flebitis infecciosa $(0,20$ a 0,04 por 1.000 camas ocupadas; $-80 \%$ ) sin cambios en las de tipo no infeccioso (2,3 por 1.000 camas ocupadas en ambos períodos) (Figura 3). Así, el programa de intervención tuvo un impacto significativo en la tasa de eventos infecciosos (Tabla 2; OR 0,17, IC 95\%: 0,34-0,80; p: 0,02). La exclusión de pacientes con más de un evento no cambió este impacto (OR 0,18; IC 95\% 0,36-0,85; p: 0,02).

\section{Cumplimiento de las medidas preventivas}

Durante la etapa de evaluación se efectuó una supervisión con pautas de los diferentes componentes enseñados en la etapa de intervención. Estos resultados fueron comparados con una medición similar efectuada en el año 2013 (Tabla 3). El cumplimiento para cada uno de los 
componentes preventivos mostró un incremento que fue significativo en el caso del registro escrito de la instalación de un CVP y en el porcentaje con cumplimiento total.

\section{Discusión}

La flebitis es habitualmente una inflamación causada por una combinación de factores químicos y mecánicos y es reconocido que la mayor parte de ellas no son infec$\operatorname{ciosas}^{21,22}$. Aparte de las bacteriemias asociadas a CVP, no tenemos conocimiento de una definición para eventos infecciosos que permita discriminarlas de aquellas de causa irritativa o traumática. En este trabajo aplicamos dos criterios para capturar esta posibilidad: bacteriemia y pus en el sitio de inserción. Ambos han sido descritos en la literatura médica asociados a flebitis séptica ${ }^{21,23-25}$ y parece ser que es importante separar las irritantes/traumáticas de las de origen infeccioso por varias razones.

Nuestros resultados sugieren que las flebitis infecciosas son más graves y se asocian a complicaciones y mortalidad respecto a las otras. Además, parecen responder más claramente a programas de intervención y se desarrollan más tarde, indicando probablemente una manipulación inapropiada del CVP. Asimismo, no parecen estar relacionadas a compuestos irritantes, sugiriendo nuevamente que el cuidado inapropiado, participa en su génesis. Finalmente, se asocian con mayor frecuencia a fiebre o con la combinación de fiebre e hipotensión arterial, fenómenos que podrían ayudar en su reconocimiento. En conjunto, estas características sugieren separar los eventos de flebitis asociada a CVP en al menos dos grupos. Ello porque probablemente tienen diferentes mecanismos patogénicos y estrategias preventivas. Ciertamente, algunos eventos en el grupo no infeccioso podrían representar infecciones localizadas y auto-limitadas. Sin embargo, ello no parece ser relevante en términos de complicaciones o mortalidad y los médicos a cargo, rara vez prescriben antimicrobianos para su tratamiento.

Este trabajo demuestra que las estrategias de prevención tuvieron un gran impacto para disminuir las flebitis infecciosas, pero sólo una fracción del total se redujo, manteniendo el discomfort sobre el paciente. En el lado positivo, los eventos graves y letales fueron prevenidos $y$ los resultados se obtuvieron en un corto plazo. No podemos anticipar si en el largo plazo el impacto podría haber involucrado las flebitis no infecciosas, pero un estudio español logró demostrar un impacto luego de varios años en las tasas globales de flebitis (de 4,8 a 1,8\%) aunque sin separarlas en infecciosas o no infecciosas ${ }^{3}$. Como en otras experiencias, la combinación de capacitación y supervisión tuvo resultados positivos en el control de infecciones ${ }^{26,27}$. Usamos sesiones de capacitación breves y repetidas tal como ha sido recomendado debido a su impacto favorable ${ }^{28}$. Esta estrategia permite contrarrestar los bajos porcentajes de conocimiento que tiene el personal de salud respecto a medidas preventivas en accesos vasculares $^{29}$.

En la intervención incluimos medidas de prevención de rutina ya conocidas por el equipo de salud y se reforzaron para homogeneizar prácticas. Nuestro sistema de capacitación permitió una retroalimentación inmediata y la creación de una cultura de prevención que ha demostrado contribuir a la reducción sostenida de infecciones nosocomiales debido a un mejor cumplimiento de las prácticas de atención ${ }^{30}$. La retroalimentación parece ser más eficiente cuando involucra a un supervisor o colega y cuando es repetitiva ${ }^{31,32}$. En nuestro caso, esta retroalimentación fue desarrollada por el personal del equipo de IAAS. Otras experiencias han ocupado medios audiovisuales, por ejemplo, durante la introducción de desinfectantes específicos (alcohol-clorhexidina) para prevenir infecciones del torrente sanguíneo asociadas a catéter ${ }^{26}$.

No observamos impacto en la forma más común de flebitis, las denominadas no infecciosas. Este nulo efecto puede estar relacionado al uso persistente de compuestos irritantes a través del CVP con diluciones insuficientes o en períodos cortos de tiempo, trauma o fragilidad endotelial o quizás otros factores. Sólo recientemente se está estandarizando la dilución y tiempos de infusión de medicamentos y haciendo esfuerzos, además, para restringir la nutrición parenteral a través de CVP, junto al desarrollo de un equipo de terapia endovenosa que esperamos pueda impactar en la frecuencia global de las flebitis asociadas a CVP. En nuestro hospital, sólo se usan CVP de poliuretano que se asocian a menos flebitis que otros materiales ${ }^{22}$, no aplicamos gel antiinflamatorio local y el lumen del catéter es uniforme ${ }^{20}$, de un diámetro no asociado a un mayor riesgo de flebitis ${ }^{33,34}$. Por otra parte, sólo se autorizan conectores sin aguja específicos, descartando aquellos de diseño interior con intrincado, los que se asocian a una alta tasa de bacteriemias ${ }^{11-15,35}$.

Este trabajo tiene varias limitaciones que deben ser mencionadas. En primer lugar, los datos fueron obtenidos a través de un sistema de vigilancia pasiva con la posible omisión de algunos eventos. No obstante, la tasa de reportes aumentó luego de la etapa de intervención sugiriendo que la vigilancia pasiva e información mejoraron. La tasa estimada de bacteriemias por CVP es de 0,1 por 100 CVP instalados y de 0,5 por 1.000 días de exposición ${ }^{25}$, pero lamentablemente nuestro diseño impide calcular tasas de incidencia.

Además, se aplicó múltiples componentes en forma simultánea, impidiendo saber la relevancia específica de cada uno de ellos. Por otra parte, no todos los eventos asignados al grupo no infeccioso fueron estudiados con hemocultivos, un examen que quedó supeditado a la decisión del médico a cargo. Así, es posible que alguno de los cuatro de los 11 eventos con fiebre en el grupo no 
infeccioso, que no fueron estudiados con hemocultivos, haya cursado con bacteriemia. Sin embargo, la asignación de estos cuatro casos al grupo con flebitis infecciosa no altera el impacto del programa, subrayando la importancia de nuestros hallazgos.

Se describe que la colocación el CVP en el lado ventral del antebrazo, en la muñeca o en las piernas se asocia a un mayor riesgo de flebitis respecto a un CVP posicionado en el lado dorsal del antebrazo o mano, pero nuestro diseño no exploró esta variable ${ }^{21,22}$.

En conclusión, nuestro programa de prevención de flebitis infecciosas, arbitrariamente definidas como aquellas asociadas a bacteriemia o pus local, tuvo un impacto favorable. Los eventos de flebitis infecciosa fueron relevantes e incluyeron casos con endocarditis o shock séptico y uno de los 10 eventos en este grupo falleció por esta causa. Cabe destacar que estos dos casos graves se asociaron a $S$. aureus y se produjeron en la etapa pre intervención.

Los eventos de flebitis infecciosa se asociaron significativamente a fiebre o a fiebre e hipotensión arterial diastólica y aparecieron más tardíamente que los eventos no infecciosos.

La distinción en dos grupos opuestos parece ser relevante debido a que los de naturaleza infecciosa se asocian a complicaciones y mortalidad, responden a programa de intervención, son más tardías y no se asocian al uso de compuestos irritantes, sugiriendo que la manipulación inapropiada participa en su patogenia. Desafortunadamente, sólo una fracción de las flebitis puede ser prevenida a través del enfoque convencional de prevención de infecciones, dado que para reducir las flebitis químicas se requiere una intervención específica que apunte a ese mecanismo en particular, que incluya diluciones de medicamentos adecuadas, tiempos adecuados de administración y/o elección de otro tipo de acceso vascular para la administración de medicamentos flebíticos, entre otras estrategias.
Agradecimientos. Agradecemos el valioso aporte de María Paz Masafierro, Enfermera Jefe del Departamento de Enfermería, Ana Gálvez, Enfermera Coordinadora de Cirugía, Gabriela Parraguéz, Enfermera Coordinadora de Medicina, Mercedes Pearcy de la Unidad de Infecciones Asociadas a la Atención de Salud y María Paz Catalán y Soraya Beltrán del Departamento de Calidad.

\section{Resumen}

Introducción: No hay criterios establecidos para distinguir las flebitis de causa infecciosa de las no infecciosas. Objetivo: Evaluar el impacto de un programa de intervención en la tasa de flebitis de tipo infecciosa (asociada a bacteriemia sin otro foco o pus local) y no infecciosa (el resto). Pacientes y Métodos: Estudio intervencional en tres etapas: diagnóstico de situación basal, intervención y evaluación. Resultados: Se detectaron 10 casos de flebitis infecciosa y 186 no infecciosas. Las flebitis infecciosas disminuyeron con la intervención $(0,2$ a 0,04 eventos por 1.000 días camas; $p=0,02)$ pero no las no infecciosas (2,3 a 2,3 por 1.000 días camas). Cinco casos con flebitis infecciosa tuvieron bacteriemia, uno de ellos con endocarditis y reemplazo valvular y otro con shock séptico y desenlace fatal. Ninguno de los casos en el grupo no infeccioso se complicó o falleció. Las flebitis infecciosas se presentaron más tarde que las otras $(4,1$ versus 2,4 días; $p=0,007)$ y se asociaron a fiebre (40\% vs $5,9 \%, p=0,004)$. Las flebitis no infecciosas se asociaron al uso de compuestos irritantes (OR 6,1; $\mathrm{IC}_{95}$ 1,3-29, $\mathrm{p}<0,05)$. Conclusiones: El programa demostró un impacto favorable para disminuir sólo las flebitis de tipo infecciosa. La distinción parece ser relevante porque las infecciosas se asocian a fiebre, complicaciones o letalidad, parecen responder a programas de prevención y son más tardías.

\section{Referencias bibliográficas}

1.- Rosenthal K. When your patient develops phlebitis. Nursing 2006; 36: 14.

2.- Jackson A. Infection control-a battle in vein: infusion phlebitis. Nurs Times 1998 (3); 94: 68-71.

3.- Ferrete-Morales C, Vásquez-Pérez M A, Sánchez-Berna M, Gilabert-Cerro I, CorzoDelgado J E, Pineda-Vergara J A, et al. Incidence of phlebitis due to peripherally inserted venous catheters: impact of a catheter management protocol. Enferm Clin 2010; 20: 3-9.

4.- Tager I, Ginsberg M, Ellis S, Walsh N, Dupont I, et al. An epidemiologic study of the risks associated with peripheral intravenous catheters. Am J Epidemiol 1983; 118: 839-51.

5.- Cornely O, Bethe U, Pauls R, Waldschmidt D. Peripheral Teflon catheters: factors determining incidence of phlebitis and duration of cannulation. Infect Control Hosp Epidemiol 2002; 23: 249-53.

6.- Singh R, Bhandary S, Pun K D. Peripheral intravenous catheter related phlebitis and its contributing factors among adult population at KU Teaching Hospital Kathmandu Univ Med J (KUMJ) 2008; 6: 443-7.

7.- O'Grady N, Alexander M, Burns L, Dellinger P, Garland J, Heard S, et al. Guidelines for the Prevention of Intravascular Catheter-Related Infections, CDC, http://www.cdc.gov/hicpac/ pdf/guidelines/bsi-guidelines-2011.pdf; 2011 [último acceso 24 de septiembre de 2016].

8.- Garitano B, Barberena C, Alonso M, Gistau C. Revisión sistemática: Efectividad de los cuidados en el mantenimiento de catéteres de inserción periférica. Enferm Clin 2002; 12: 166-72.

9.- Regueiro Pose M A, Souto Rodríguez B, Iglesias Maroño M, Outón Fernández I, Cambeiro Núñez J, Pértega Díaz S, et al. Peripheral venous catheters: incidence of phlebitis and its determining factors. Rev Enferm 2005; 28: 21-8.

10.- Martínez J A, Piazuelo M, Almela M, Blecua $\mathrm{P}$, Gallardo R, Rodríguez S, et al. Evaluation of add-on devices for the prevention of phlebitis 
and other complications associated with the use of peripheral catheters in hospitalised adults: a randomised controlled study. J Hosp Infect 2009; 73: 135-42.

11.- Jarvis W, Murphy C, Hall K, Fogle P, Karchmer $\mathrm{T}$, Harrington G, et al. Health care-associated bloodstream infections associated with negative -or positive-pressure or displacement mechanical valve needleless connectors. Clin Infect Dis 2009; 15; 49: 1821-7.

12.- Btaiche I, Kovacevich D, Khalidi N, Papke L. The effects of needleless connectors on catheter-related bloodstream infections. Am J Infect Control 2011; 39: 277-83.

13.- Mazher M, Kallen A, Edwards J, Donlan R. An in vitro evaluation of disinfection protocols used for needleless connectors of central venous catheters. Lett Appl Microbiol 2013; 57: 282-7.

14.- Donlan R, Murga R, Bell M, Toscano C, Carr J, Novicki T, et al. Protocol for detection of biofilms on needleless connectors attached to central venous catheters. J Clin Microbiol 2001; 39: 750-3.

15.- Pérez E, Williams M, Jacob J, Reyes M, Chernetsky Tejedor S, Steinberg J, et al. Microbial biofilms on needleless connectors for central venous catheters: comparison of standard and silver-coated devices collected from patients in an acute care hospital. J Clin Microbiol 2014; 52: 823-31.

16.- Drugs that cause phlebitis. In: DRUGDEX ${ }^{\circledR}$ System (electronic version). Truven Health Analytics, Greenwood Village, Colorado, USA 2016. Available at: http://www. micromedexsolutions.com/ [Accedido 19 de enero de 2016].

17.- Pérez E. Post infusión phlebitis in peripheral venous catheters: an actualization of the art. Horiz Enferm http://revistahorizonte.uc.cl/ images/pdf/22-2/flebitis_postinfusion_en_ cateteres.pdf; 2011 [último accedido el 24 de septiembre de 2016].

18.- Adesami O, Stranz M. Infusion Drug List: $\mathrm{pH}$, osmolality, reported phlebitis. http:// www.ctins.org/Marc\%20Stranz\%20Drug\%20 Chart\%20pH\%20Osmol\%202003.pdf; 2003 [último accedido 24 de septiembre de 2016].

19.- Milutinović D, Simin D, Zec D. Risk factors for phlebitis: a nurse perception obtained by a questionnaire. Rev Lat-Am Enfermagem 2015; 23: 677-84.

20.- Arrazola Saniger M, Lerma García D, Ramírez Arrazola A. The most common complications of intravenous drug administration: phlebitis and extravasation. Enfermería Clínica 2012; 12 : 80-5.

21.- van der Sar-van der Brugge S, Posthuma E F. Peripheral intravenous catheterrelated phlebitis. Ned Tijdschr Geneeskd 2011; 155: A3548.

22.- Maki D G, Ringer M. Risk factors for infusionrelated phlebitis with small peripheral venous catheters. A randomized controlled trial. Ann Intern Med 1991; 114: 845-54.

23.- Espinoza R, Andresen M, Nervi B, Rosemberg H. Septic phlebitis: origin of severe septic condition. Rev Med Chile 1998; 126: $195-8$.

24.- Hong S K, Nam S H, Kim H C. Fatal peripheral candidal suppurative thrombophlebitis in a postoperative patient. J Korean Med Sci 2008; 23: 1094-6.

25.- Maki D G, Kluger D M, Crnich C J. The risk of bloodstream infection in adults with different intravascular devices: a systematic review of 200 published prospective studies. Mayo Clin Proc 2006; 81: 1159-71.

26.- Sannoh S, Clones B, Muñoz J, Montecalvo M, Parvez B. A multimodal approach to central venous catheter hub care can decrease catheterrelated bloodstream infection. Am J Infect Control 2010; 38: 424-9

27.- Sjöberg M, Eriksson M. Hand disinfectant practice: the impact of an education intervention. Open Nurs J 2010; 23; 4: 20-4.

28.- Flodgren G, Conterno L, Mayhew A, Omar O, Pereira C R, Shepperd S, et al. Interventions to improve professional adherence to guidelines for prevention of device-related infections. Cochrane Database Syst Rev 2013; 3 : CD006559.

29.- Cicolini G, Simonetti V, Comparcini D, Labeau S, Blot S, Pelusi G, et al. Nurses knowledge of evidence-based guidelines on the prevention of peripheral venous catheter-related infections: a multicentre survey. J Clin Nurs 2014; 23: 257888.

30.- Lobo R, Levin A, Oliveira M, Gomes L, Gobara, Park M, et al. Evaluation of interventions to reduce catheter-associated bloodstream infection: continuous tailored education versus one basic lecture. Am J Infect Control 2010; 38: 440-8.

31.- Mauger B, Marbella A, Pines E, Chopra R, Black E, Aronson N, et al. Implementing quality improvement strategies to reduce healthcare-associated infections: A systematic review. Am J Infect Control 2014; 42: S274-83.

32.- Ivers N, Jamtvedt G, Flottorp S, Young J, Odgaard-Jensen J, French S, et al. Audit and feedback: effects on professional practice and healthcare outcomes. Cochrane Database Syst Rev 2012; 6: CD000259.

33.- Payne-James J, Bray M, Kapadia S, Rana S, Mcswiggan D, Silk D. Topical nonsteroidal anti-inflammatory gel for the prevention of peripher a vein thrombophlebitis. Anaesthesia 1992; 47: 324-6.

34.- Khawaja H, Payne-James J. Survival of peripheral intravenous infusions. Br Med J 1992 18; 304: 1053-4.

35.- Blake M. Update: Catheter-related bloodstream infection rates in relation to clinical practice and needleless device type. Can J Infect Control 2008; 23: 156-60. 